\title{
Variación antigénica de la cepa Munantá de Trypanosoma cruzi después de pase por ratón
}

\author{
Lilian A. Santana ${ }^{1}$, Marleny Montilla ${ }^{2}$, Santiago Nicholls ${ }^{2}$, Concepción J. Puerta ${ }^{1}$
}

\begin{abstract}
Resumen
Este estudio evaluó los cambios en el perfil isoenzimático y antigénico de la cepa Munantá de Trypanosoma cruzi después de dos pases consecutivos por ratón, mediante electrofóresis isoenzimática, electrofóresis en PAGE-SDS e immunoblot. Los resultados obtenidos muestran diferencias estadísticamente significativas entre la cepa antes y después de los pases en los animales, lo cual sugiere que el ratón no es un modelo recomendable para obtener las formas tripomastigotas de la cepa Munanta del parásito destinadas a estudios de la respuesta inmune.
\end{abstract}

\section{Antigenic variation in Trypanosoma cruzi Munanta strain after passages in mice}

This study evaluated the isoenzyme and antigenic changes of Trypanosoma cruzis Munanta strain after two consecutive passages in mice, using isoenzyme electrophoresis, SDS-PAGE electrophoresis and immunoblot. The results obtained show significant statistical differences within the strain before and after animal passages, which suggests that the mouse is not a recommended model for obtaining the parasite's Munanta strain's trypomastigote forms destined for study of the immune response.

La tripanosomiosis americana o enfermedad de Chagas es producida por Trypanosoma cruzi, un protozoo flagelado de la familia Trypanosomatidae y transmitida por insectos hematófagos de los géneros Rhodnius, Triatoma y Panstrongylus, de los cuales Rhodnius prolixus es el más significativo en Colombia.

La enfermedad de Chagas presenta alta incidencia en Latinoamérica, donde se estima que afecta a más de 15 millones de personas (1). En Colombia, la enfermedad se encuentra distribuida en las zonas rurales del nordeste del país (cuenca del Catatumbo), Magdalena Medio, Boyacá, Tolima, Cundinamarca, Huila y Llanos Orientales (piedemonte, La Macarena y Meta).
Trypanosoma cuzi es un parásito con un complejo ciclo de vida el cual se desarrolla en el insecto vector y en el huésped mamífero, presentando diversos estadios morfológicos de desarrollo (2), de los cuales, la forma tripomastigota reviste gran importancia ya que, además de ser la forma infectante, se encuentra en contacto con el sistema inmune del huésped.

Para aislar los tripomastigotes, se ha recurrido a simular el ciclo de vida del parásito, bien sea utilizando animales de laboratorio como el ratón o por medio de cultivo in vitro en células. No obstante, Da Silva et al. (1989) informan que existen diferencias significativas en el perfil antigénico de los tripomastigotes sanguíneos

\footnotetext{
1 Departamento de Microbiología, Facultad de Ciencias, Pontificia Universidad Javeriana, Santa Fe de Bogotá, Colombia.

2 Laboratorio de Parasitología, Instituto Nacional de Salud, Santa Fe de Bogotá, Colombia.

Recibido para su publicación: 22 de abril de 1998. - Aprobado para su publicación: 1 de mayo de 1998.
} 
aislados de ratón y de los tripomastigotes obtenidos en cultivos de células LLC-MK2.

Diferentes estudios demuestran cómo el pase de $T$. cruzi en ratones condiciona el perfil isoenzimático del parásito $(4,5)$. Así mismo, el ratón puede entonces contribuir a las diferencias encontradas entre los tripomastigotes sanguíneos y los tripomastigotes derivados de cultivo tisular.

Dentro del anterior contexto, en este estudio se evaluó el perfil antigénico de la cepa colombiana Munantá de T. cruzi antes y después de dos pases consecutivos en ratón, mediante PAGESDS e immunoblot.

\section{Materiales y métodos}

Parásitos. La cepa Munantá de T. cruzi, aislada a partir de vectores de la vereda de Munantá en el municipio de Guateque en Boyacá, fue suministrada por el Grupo de Parasitología del Instituto Nacional de Salud. Los parásitos fueron descongelados y cultivados en medio NNN y Maekelt a $24^{\circ} \mathrm{C}(6)$.

Infección en ratones. Se utilizaron ratones $\mathrm{Balb} / \mathrm{C}$ de 15 a 20 días de edad, los cuales fueron inoculados intraperitonealmente con parásitos mantenidos durante 20 días en cultivo. Una vez obtenida una alta parasitemia, aproximadamente 1 mes, se sangraron los ratones y se hizo un repique a un segundo grupo de ratones de las mismas caraterísticas.

Infectividad. Los ratones fueron revisados semanalmente haciendo un pequeño corte en el final de la cola, obteniéndose una gota de sangre para la preparación en fresco, la cual se revisó al microscopio de luz para visualizar los parásitos.

Recuperación de los parásitos. Una vez detectado el pico de parasitema ( 1 mes en el primer pase y 8 días en el segundo pase), los ratones fueron sangrados directamente del corazón; la sangre $(0,1 \mathrm{~mL})$ fue llevada a medio de cultivo NNN para la recuperación de los parásitos y, posteriormente, a Maekelt (6).

Caracterización isoenzimática. Los cultivos de los parásitos en fase logarítmica de crecimiento, se lavaron tres veces con PBS $\left(\mathrm{NaH}_{2} \mathrm{PO}_{4}\right.$ $\left.0,003 \mathrm{M}, \mathrm{Na}_{2} \mathrm{HPO}_{4} 0,007 \mathrm{M}, \mathrm{NaCl} 0,13 \mathrm{M} \mathrm{pH} 7,2\right)$ a $4000 \mathrm{~g}, 4{ }^{\circ} \mathrm{C}$, durante $10 \mathrm{~min} y$ se resuspendieron en solución salina al $0,85 \%$ a una concentración de $1 \times 10^{6}$ a $1 \times 10^{7}$ parásitos/ $\mathrm{mL}$. Posteriormente, los parásitos fueron sometidos a lisis osmótica en un estabilizador enzimático (DTT $2 \mathrm{mM}$, ácido E-aminocaproico $2 \mathrm{mM}$ y EDTA $2 \mathrm{mM}$ ), durante $10 \mathrm{~min}$ a $4 \stackrel{\circ}{\circ} \mathrm{C} \mathrm{y}$ centrifugados a $8000 \mathrm{~g}, 4 \stackrel{\circ}{\circ} \mathrm{C}$, durante $15 \mathrm{~min}$ (7). El sobrenadante recuperado, denominado extracto enzimático, fue almacenado a $-70 \stackrel{\circ}{\circ}$. Las isoenzimas de los extractos fueron separadas posteriormente mediante electrofóresis en capa delgada de acetato de celulosa, de acuerdo con lo descrito por Miles et al. (1980) y S.B. Abderrazak et al. (1993).

Los sistemas enzimáticos estudiados fueron: malato deshidrogenasa (MDH) E.C.1.1.1.40, isocitrato deshidrogenasa (IDH) E.C. 1.1.1.42, glucosa fosfato isomerasa (GPI) E.C.5.3.1.9, peptidasa 1 (PEP-1) E.C.3.4.11*, peptidasa 2 (PE) E.C.3.4.11*, glucosa-6-fosfato deshidrogenasa (G6PD) E.C.1.1.1.49, glutamato deshidrogenasa NAD/NADP (GDH) E.C.1.4.1.3., enzima málica (ME) E.C.1.1.1.4.0, fosfoglucomutasa (PGM) E.C.2.7.5.1, 6-fosfogluconato deshidrogenasa (6PGDH) E:C:1.1. 1.44 y aspartato aminotransferasa (GOT) E.C.2.6.1.1.

Separación electroforética. Los parásitos fueron recolectados por centrifugación a $900 \mathrm{~g}$ a $4^{\circ} \mathrm{C}$ y lavados con PBS. Posteriormente, éstos fueron contados en cámara de Neubauer y resuspendidos en solución de lisis (Tris $50 \mathrm{mM}$, EDTA $5 \mathrm{mM}$, Bisulfito de sodio $380 \mathrm{ng} / \mathrm{mL}$, NP40 1\%, SDS 1\%, PMSF $1 \mathrm{mM}$, aprotinina $0,5 \mathrm{mg} / \mathrm{mL}$ ) a una concentración de $5 \times 10^{5}$ parásitos $/ \mathrm{mL}$.

Una vez resuspendidos, los parásitos se sometieron a diez ciclos de congelación y descongelación en nitrógeno líquido a $-70^{\circ} \mathrm{C}$ y baño serológico a $37^{\circ} \mathrm{C}$, respectivamente. $\mathrm{Pa}$ sado este proceso, se centrifugaron a $4.000 \mathrm{~g}$, $4^{\circ} \mathrm{C}$; se extrajo la fracción proteica de cada lisado y se hicieron alícuotas que se almacenaron $\mathrm{a}-70^{\circ} \mathrm{C}$. 
Posteriormente, las proteínas presentes en los lisados de las cepa Munantá de T. cruzi antes $\left(N_{0}\right)$ y después de dos pases por ratón $\left(N_{2}\right)$ fueron cuantificadas según el método de Bradford (10) y separadas mediante electrofóresis en geles de poliacrilamida según el protocolo de Laemli (11), utilizando una concentración de lisado de $12 \mathrm{mg}$ por pozo corrido. Las bandas resueltas fueron visualizadas mediante tinción con azul de Coomassie y tinción de plata (12). Los geles fueron conservados y secados en secador eléctrico a $51^{\circ} \mathrm{C}$ durante 13 horas.

Inmunodetección. Las fracciones proteicas de los lisados $\left(\mathrm{N}_{0}\right.$ y $\left.\mathrm{N}_{2}\right)$ fueron transferidas a papel de nitrocelulosa (13), coloreadas con Ponceau S y detectadas inmunológicamente, utilizándose un pool de sueros chagásicos crónicos (276/ $95,275 / 95$ procedentes de Cundinamarca y 265/95, 266/95, 267/95, procedentes de Casanare) como primer anticuerpo y anti-lgG humana acoplada a peroxidasa (Sigma), como segundo anticuerpo.

\section{Resultados}

Perfill isoenzimático. De los nueve sistemas isoenzimáticos analizados, uno de ellos, GDH, varió tras el pase de la cepa Munantá por ratón. Es así como en la electrofóresis para dicha enzima, se observaron dos bandas antes de los pases de la cepa por ratón, mientras que luego del primero y segundo pase, se observó tan solo una banda (figura 1).

Perfill antigénico. La concentración de proteínas según el método de Bradford fue de 1,2 mg/ $\mathrm{mL}$ para $\mathbb{N}_{0}$ (lisado sin pases por ratón) y 2,4

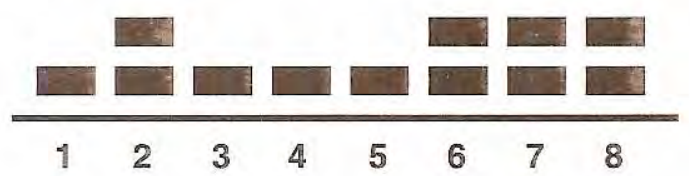

Figura 1. Representación esquemática del patrón isoenzimático de la GDH de la cepa Munantá de T. cruzi antes y despúes de su pase por ratón, usando las cepas F. Chaparro, Tulahuén, MDID \#2, MDID 409 y MDID R-27, como referencia. 1: F. Chaparro; 2: Munantá antes de pase por ratón; 3: Munantá despúes del primer pase por ratón; 4: Munantá despúes del segundo pase por ratón; 5 : Tulahúen; 6: MDID \#2; 7: MDID 409, y 8: R-27. $\mathrm{mg} / \mathrm{mL}$ para $\mathrm{N}_{2}$ (lisado con dos pases por ratón). Una vez corrido el gel, éste se dividió en dos, una parte se reveló con tinción de plata y la otra con tinción de Coomassie. Para el análisis de resultados, se tuvo en cuenta la tinción de plata. por ser más sensible (figuras 2 y 3 ). Las fracciones resueltas se presentan en el cuadro 1.

El análisis estadístico realizado con el programa EPI-INFO mostró diferencias estadísticamente significativas, para $p<0,05$, entre las fracciones

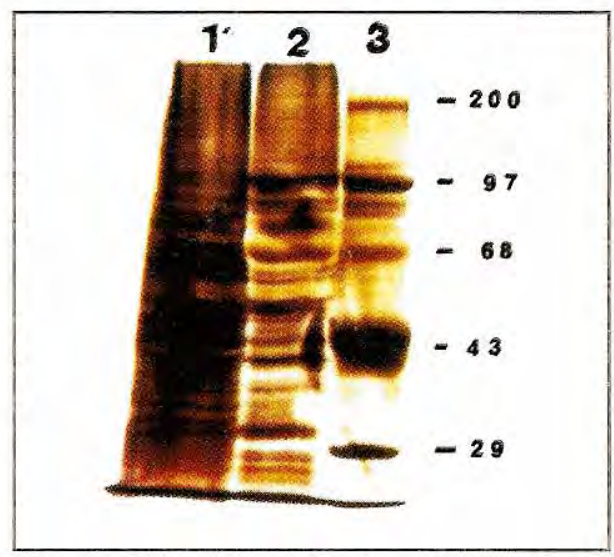

Figura 2. Page-SDS de lisado de epimastigotes de la cepa Munantá de T. cruzi, revelada con tinción de plata. 1) $\mathrm{N}_{2}$ : lisado despúes de dos pases por ratón y 3) patrones de peso molecular (Kd).

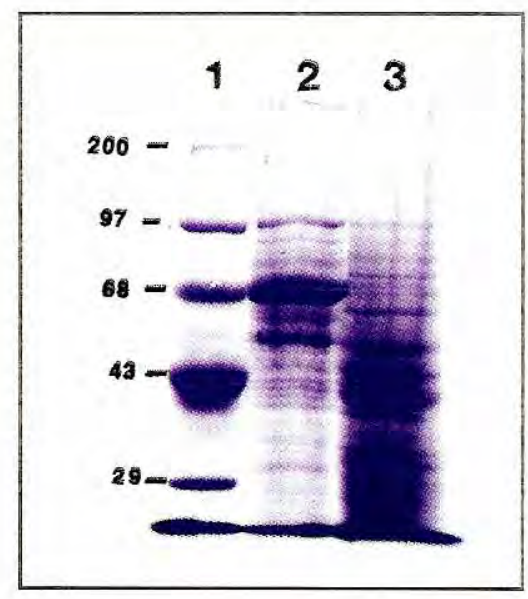

Figura 3. Page-SDS de lisados de epimastigotes de la cepa Munantá de T. cruzi, revelada con tinción de azul de Coomassie. 1) Patrones de peso molecular (Kd); 2) $N_{0}$ : lisado antes de pases por ratón, y 3) $\mathrm{N}_{2}$ : lisado despúes de dos pases por ratón. 
Cuadro 1. Fracciones proteícas de la cepa munanta de T. Cruzi obtenidas mediante PAGE-SDS, antes y después de dos pases por ratón.

\begin{tabular}{|c|c|c|}
\hline PM (Kd) & no & $\mathrm{N}_{2}$ \\
\hline 23 & $x$ & \\
\hline 23,5 & $x$ & $x$ \\
\hline 24,5 & $x$ & $x$ \\
\hline 25,5 & $x$ & \\
\hline 28 & & $x$ \\
\hline 29 & $x$ & $x$ \\
\hline 30 & & $x$ \\
\hline 31 & $x$ & \\
\hline 33,5 & $x$ & $x$ \\
\hline 34 & $x$ & \\
\hline 35 & $x$ & $x$ \\
\hline 36 & & $x$ \\
\hline 37 & $x$ & $x$ \\
\hline 41,5 & $x$ & $x$ \\
\hline 42 & $x$ & $x$ \\
\hline 43 & $x$ & $x$ \\
\hline 45 & & $x$ \\
\hline 46 & $x$ & $x$ \\
\hline 48 & $x$ & \\
\hline 50 & $x$ & $x$ \\
\hline 51 & & $x$ \\
\hline 55 & & $x$ \\
\hline 58 & $x$ & $x$ \\
\hline 59 & $x$ & $x$ \\
\hline 65 & & $x$ \\
\hline 69 & $x$ & $x$ \\
\hline 75 & & $x$ \\
\hline 75,5 & & $x$ \\
\hline 79 & $x$ & \\
\hline 80 & & $x$ \\
\hline 82 & $x$ & $x$ \\
\hline 87 & $x$ & $x$ \\
\hline 92 & $x$ & \\
\hline 95 & $x$ & \\
\hline 97 & $x$ & $x$ \\
\hline 105 & $x$ & $x$ \\
\hline 108 & $x$ & \\
\hline 110,5 & $x$ & $x$ \\
\hline 120 & $x$ & $x$ \\
\hline 130 & $x$ & $x$ \\
\hline 140 & $x$ & $x$ \\
\hline 145 & $x$ & \\
\hline 150 & $x$ & \\
\hline 160 & $x$ & $x$ \\
\hline 180 & $x$ & $x$ \\
\hline 190 & $x$ & $x$ \\
\hline 210 & $x$ & \\
\hline 240 & $x$ & \\
\hline 250 & $x$ & \\
\hline 330 & $X$ & \\
\hline
\end{tabular}

proteicas antes y después de dos pases consecutivos por ratón.

Análisis posteriores demostraron que el $50 \%$ de las fracciones proteicas se conserva después de los pases por ratón y el $50 \%$ restante cambia, dentro del cual $33 \%$ de las fracciones proteicas desaparece después de los pases y $17 \%$ aparece tras dos pases consecutivos por ratón. El análisis desarrollado sobre la distribución del número de fracciones proteicas según el rango de peso molecular, demuestra que las diferencias significativas se presentan en el rango de 98-350 $\mathrm{Kd}$, presentándose un doble número de fracciones antes de los pases que después. El mayor número de fracciones proteicas compartidas se encuentra distribuido en el rango de 30 a $43 \mathrm{Kd}$, mientras que el rango de 44 a $68 \mathrm{Kd}$ presenta el menor número de fracciones proteicas conservadas.

Adicionalmente, se analizaron los cambios en la intensidad de las fracciones proteicas resueltas, observándose que los cambios en las intensidades de las fracciones proteicas ocurren independientemente de su peso molecular; sin embargo, en el rango de 44 a $68 \mathrm{Kd}$ se encontraron la mayoría de las fracciones proteicas intensas que se conservaron, seguidas por el rango de 69 a $97 \mathrm{Kd}$. Por otro lado, $40 \%$ de las fracciones proteicas más intensas desaparecen tras los dos pases sucesivos en ratón, mientras que $12 \%$ de las fracciones proteicas intensas aparecen o se encuentran sólo tras los pases por ratón.

En cuanto a las fracciones resueltas en gel y transferidas a papel de nitrocelulosa para el reconocimiento inmunológico con sueros de pacientes chagásicos crónicos, se obtuvo un menor número de fracciones proteicas reconocidas según se presenta en el cuadro 2 .

Los resultados de la prueba $t$ de Student realizada, muestran diferencias estadísticamente significativas entre las fracciones proteicas reconocidas por los sueros chagásicos antes y después de los pases por ratón, para una $\mathrm{p}<0,1$.

Los análisis realizados sobre el comportamiento de las fracciones reconocidas, demuestran que 
Tabla 2. Reconocimiento inmune de las fracciones proteicas de la cepa Munanta de T. Cruzi obtenida antes y después de dos pases por ratón.

\begin{tabular}{ccc}
\hline PM (Kd) & No & $N^{\text {N }}$ \\
\hline 38 & $\mathrm{X}$ & \\
39 & $\mathrm{X}$ & $\mathrm{X}$ \\
50 & & $\mathrm{X}$ \\
60 & & $\mathrm{X}$ \\
67 & & $\mathrm{X}$ \\
70 & & \\
75 & $\mathrm{X}$ & \\
80 & $\mathrm{X}$ & $\mathrm{X}$ \\
87 & & $\mathrm{X}$ \\
95 & $\mathrm{X}$ & \\
100 & $\mathrm{X}$ & \\
105 & $\mathrm{X}$ & \\
108 & $\mathrm{X}$ & \\
110 & $\mathrm{X}$ & $\mathrm{X}$ \\
145 & $\mathrm{X}$ & $\mathrm{X}$ \\
150 & & \\
\hline
\end{tabular}

$31 \%$ de las fracciones se conserva tras los pases por ratón, mientras $38 \%$ sólo es reconocida previo al pase y $31 \%$ corresponde a nuevas fracciones reconocidas tras los pases por ratones. Independientemente de si el mayor número de fracciones proteicas fueron reconocidas antes o después de los pases por ratón, el mayor número de fracciones reconocidas se ubica en el rango de 97 a $350 \mathrm{Kd}$, seguido por el rango de 67 a $97 \mathrm{Kd}$. Finalmente, se observó una correlación entre los resultados obtenidos por PAGE-SDS e immunoblot.

\section{Discusión}

Los resultados mostrados anteriormente revelan cómo el pase sucesivo de la cepa Munantá de T. cruzi por ratones adultos afecta significativamente no sólo el patrón de corrido electroforético de sus proteínas, sino el reconocimiento de las mismas por un pool de sueros de pacientes chagásicos crónicos, sugiriendo entonces un cambio en el perfil antigénico que, a su vez, se acompaña de variaciones en el perfil isoenzimático.

Llamativamente, Da Silva et al. (1989) informan en su estudio que las principales diferencias encontradas entre los tripomastigotes sanguíneos y los derivados de cultivo tisular, es que el reconocimiento inmune de sueros chagásicos crónicos de las bandas localizadas en las regiones de 85 y $52 \mathrm{Kd}$ es mucho más débil en los tripomastigotes sanguíneos, mientras que la región de alto peso molecular presenta un mayor grado de reconocimiento en los mismos. De manera similar, en este trabajo se encontró una falta de reconocimiento inmune de las bandas situadas entre 50 y $87 \mathrm{Kd}$ en los lisados de epimastigotes del parásito $\mathrm{N}_{0}$, mientras que las localizadas en la región de alto peso molecular son ampliamente reconocidas. Estos resultados sugieren entonces que las diferencias encontradas por Da Silva en el perfil antigénico de ambos tipos de tripomastigotes podrían estar dadas por el pase de los tripomastigotes sanguíneos por ratón.

O'Daly et al. (1990), por su parte, han realizado estudios similares de variación en el perfil antigénico de $T$. cruzi, al someter cultivos de parásitos a diferentes temperaturas (14), encontrando diferencias entre los antígenos de superficie de parásitos cultivados a $30{ }^{\circ} \mathrm{C}$ y a $34^{\circ} \mathrm{C}$. Sugiriendo, entonces, cómo los cambios ambientales sufridos por los parásitos, sean éstos pase por ratón o cambio de temperatura del medio de cultivo, afectan el perfil antigénico de los mismos.

Adicionalmente, estudios longitudinales realizados por Hudson et al. (1988) muestran cómo tras 3,5 años, cinco de diecinueve pacientes cambian su perfil de reconocimiento de lisados de tripomastigotes derivados de cultivo tisular. Uno por pérdida de reconocimento de una banda de alta peso molecular y los otros por conversión de un perfil de inmunoprecipitación de seronegativos a seropositivos. Es decir que, tras su permanencia en el huésped, el parásito varía su perfil de reconocimiento antigénico.

Por su parte, los zimodemas han sido extensamente estudiados. Es así como Carneiro et al. (1990) encontraron que, tras 18 meses de mantenimiento en ratones $\mathrm{C} 3 \mathrm{H}$ (con al menos 27 pases), cinco de las trece cepas estudiadas mostraron variaciones tanto en su perfil isoenzimático como de esquizodemas. Sugiriendo dicho resultado que la población de parásitos era inicialmente mixta y, durante su pase por el ratón, se seleccionaron preferente- 
mente, las poblaciones que portaban el zimodema $\mathrm{A}$.

De igual forma, Deane et al. (1984) encontraron un efecto selectivo del ratón sobre la población de parásitos ya que al infectar ratones con dos cepas diferentes de T. cruzi, tan solo logran recuperar una sola. Así mismo, los estudios de Lauria-Pires et al. (1996) y Lauria-Pires y Texeira (1996) muestran cómo el zimodema del aislado parental hSLU239, Z3, difiere ampliamente del zimodema ZII de los clones derivados h1 y h2. Esta variación tan radical de zimodemas, sólo puede ser explicada mediante la presencia de poblaciones genéticamente heterogéneas en el aislado original.

Por otra parte, Alves et al. (1993) encontraron que clones de la cepa $Y$ cambiaban su patrón isoenzimático, de manera reversible, al pasar por ratones recién nacidos, así como también al variar los medios de cultivo y realizar procedimientos de subclonaje. Posteriormente, en 1994, dichos autores determinaron cómo los cambios observados a su vez se acompañaban de variaciones reversibles en el ADN del cinetoplasto, concluyéndose entonces que los cambios obervados obedecían a variaciones intraclonales. Así mismo, McDaniel y Dvorak (1993) encontraron cómo T. cruzi puede sufrir variaciones intraespecíficas de clon a clon.

En el presente estudio, si bien tan sólo se encontraron variaciones en un sistema enzimático, no se puede asegurar que los cambios observados se debieron a variaciones intraespecíficas. Para ello, sería necesario clonar la cepa del parásito. No obstante, se pueden señalar como factores desencadenantes de la variación antigénica e isoenzimática, los cambios en las condiciones experimentales sufridas por la cepa (pase de medio de cultivo a ratón) y los factores intrínsecos aportados tanto por la cepa y edad de los ratones (21), como por la propia cepa Munantá.

En conclusión, la recuperación de tripomastigotes de la cepa Munantá de T. cruzi bajo las condiciones aquí estipuladas, no es recomendable para trabajos en los cuales se pretenda analizar la respuesta inmune celular y/o humoral, inducida por las fracciones proteicas del parásito. Toda vez que el sistema inmune del hombre no estaría reconociendo los determinantes antigénicos e inmunogénicos que existen naturalmente en la cepa, sino aquellos seleccionados por los pases sucesivos en ratón. Finalmente, habría que tener en cuenta la extensión de la anterior observación a otras cepas del parásito.

\section{Agradecimientos}

A Luis E. Gualdrón, por su asesoría técnica en cuanto al manejo de animales de experimentación; a Martha Ayala y Sandra Corredor, por el entrenamiento recibido en el manejo de cepas y medios de cultivo y, en general, al Grupo de Parasitología del Instituto Nacional de Salud y al Departamento de Inmunología de la Pontificia Universidad Javeriana.

\section{Referencias}

1. World Health Organization. Report of WHO Expert Committee: WHO, Technical Report Series No. 811. Geneva, 1991.

2. Tanowitz HB, Kirchhoff LV, Simon D, Morris SA, Weiss LM, Wittner M. Chagas disease. Clin Microbiol Rev 1992;5:400-19.

3. Da Silva MMA, Brodskyn $\mathrm{Cl}$, Takehara HA, Mota I. Differences in the antigenic profile of bloodstream and cell culture derived trypomastigotes of Trypanosoma cruzi. Rev Instituto de Medicina Tropical de Sao Paulo1989;31:146-150.

4. Carneiro M, Chiari E, Goncalves AM, Da SilvaPereira AA, Morel CM, Romanha AJ. Changes in the isoenzyme and kinetoplast DNA patterns of Trypanosoma cruzi strains induced by maintenance in mice. Acta Tropica 1990;47:35-45.

5. Alves AMV, Tanuri A, Almeida DF, von Kruger WMA. Reversible changes in the isoenzyme electrophoretic mobility pattern and infectivity in clones of Trypanosoma cruzi. Experiment Parasitol 1993; 77:246-53.

6. Duque S, Peláez D, Corredor A. Cultivo in vitro de parásitos de la familia Trypanosomatidae. En: Normas para cultivo in vitro de parásitos de la familia Trypanosomatidae. Manual de procedimientos. Santa Fe de Bogotá: Instituto Nacional de Salud; 1993. p. 41-5.

7. Godfrey DG, Kilgour V. Enzyme electrophoresis in characterizing the causative organism of Gambia trypanosomiasis. Trans Roy Soc Trop Med Hyg 1976; 70:219-24. 
8. Miles MA, Lanham S, Sousa AA, Povoa A. Further enzymatic characters of Trypanosoma cruzi and their evaluation for strain characterization. Am J Trop Med Hyg 1980;74:221-37.

9. Abderrazak SB, Gerrini F, Daude FM, True $\mathbf{P}$, Neubauer K, Lecwicka K, Barnabe C, Tibayrenc M. Isoenzyme electrophoresis for parasite characterization. In: Hyde $\mathrm{J}$, ed. Methods in molecular biology. Volume 21: Protocols in molecular parasitology. New York; 1993. p. 361-81.

10. Bradford MM. A rapid and sensitive method for the quantification of microgram quantities of protein utilizing the principle of protein-dye binding. Ann Biochem 1976;72:248-54.

11. Laemli VK. Cleveage of structural proteins during the assembly of the head of bacteriophage T4. Nature 1970;227:680-5.

12. Merril CR, Goldman D, van Keuren ML. Gel proteins stains: silver stains methods. Meth Enzimol 1984;104:441-7.

13. Towbin H, Staehelin T, Gordon J. Electrophoretic transfer of protein from polyacrilamide gels to nitrocellulose sheets: procedure and some applications. Meth Enzimol 1979;76:4350-4.

14. O'Daly JA, Polanco N. Variability of Trypanosoma cruzi epimastigote surface antigens with changes in the temperature of the cultures. Am J Trop Med Hyg 1990;43:44-51.

15. Hudson L, Guhl F, Sánchez N, Bridge D, Jaramillo CA, Young A. Longitudinal studies of the immune response of Colombian patients infected with Trypanosoma cruzi and $T$. rangeli. Parasitology 1988;96:449-60.

16. Deane MP, Sousa MA, Pereira NM, Goncalves AM, Momen H, Morel CM. Trypanosoma cruzi: inoculation schedules ans re-isolation methods select individual strains from doubly infected mice, as demostratred by schyzodeme and zymodeme analyses. J Protozool 1984;3:276-80.

17. Laurias-Pires L, Bogliolo AR, Teixeira ARL. Diversity of Trypanosoma cruzi stocks and clones derived from Chagas disease patients. Experimen Parasitol 1996;82:182-90.

18. Laurias-Pires L, Teixeira ARL. Virulence and pathogenicity associated with diversity of Trypanosoma cruzi stocks and clones derived from Chagas disease patients. Am J Trop Med Hyg 1996;55:304-10.

19. Alves AMB, De Almedida DF, Von Kruger WMA. Changes in Trypanosoma cruzi kinetoplast DNA minicircles induced by environmental conditions and subcloning. J Eukaryotic Microbiol 1994;41:415-9.

20. McDaniel JP, Dvorak JA. Identification, isolation and characterization of naturally-ocurring Trypanosoma cruzi variants. Mol Biochem Parasitol 1993;57:22231.

21. Powell MR, Wasson DL. Host genetics and resistance to acute Trypanosoma cruzi infection in mice. I. Antibody isotype profiles. Parasite Immunol 1993;15:215-21. 